\title{
Experimental Methods for Simultaneous Measurement of Action Potentials and Electrograms in Isolated Heart
}

\author{
J. KOLÁŘOVÁ ${ }^{1}$, K. FIALOVÁ ${ }^{2}$, O. JANOUŠEK ${ }^{1}$, M. NOVÁKOVÁ ${ }^{2}$, I. PROVAZNÍK ${ }^{1}$ \\ ${ }^{1}$ Brno University of Technology, Department of Biomedical Engineering, Brno, Czech Republic, \\ ${ }^{2}$ Masaryk University, Faculty of Medicine, Physiology Department, Brno, Czech Republic
}

Received February 18, 2010

Accepted March 26, 2010

\begin{abstract}
Summary
Monophasic action potential (MAP) can be recorded from the heart surface by optical method based on fluorescence measurement. The motion of isolated heart during experiment caused additional noise in recorded signal. The motion artifact can be eliminated by ratiometric fluorescence emission measurements. This study is based on experiments in which optical MAP measurement is done by single-wavelength and dualwavelength measurement of fluorescence emission. Both recording setups are presented and their advantages and disadvantages are discussed. MAPs recorded by both methods from isolated rabbit hearts perfused according to Langendorff are presented. Simultaneous electrograms (EG) and MAPs recording are analyzed and measurement of velocity of impulse conduction through heart tissue is presented.
\end{abstract}

\section{Key words}

Isolated heart $\bullet$ Action potential $\bullet$ Electrogram $\bullet$ Voltage sensitive dye • Ratiometry

\section{Corresponding author}

J. Kolářová, Brno University of Technology, Kolejní 4, 61200 Brno, Czech Republic. E-mail: kolaraj@feec.vutbr.cz

\section{Introduction}

Heart electrophysiology is commonly studied in isolated perfused animal hearts. Experiments are aimed at description of physiological and pathophysiological states of the heart and their progression. During the experiment, various parameters under well-defined conditions may be followed, such as electrograms (EG), monophasic action potentials (MAPs), atrial and ventricular pressure, temperature, $\mathrm{pH}$, etc.

Perfusion of isolated animal heart according to Langendorff is a well established method in experimental cardiology. It has been used for more than a century and has still a very important role in basic cardiology and pharmacology research (Valentin et al. 2004). In pharmacological studies, various drugs are applied to perfused heart and the response is monitored (e.g. Novakova 2007, Novakova et al. 2008, Wall et al. 2007). The changes of electrogram yield information about heart rate changes and eventual dysfunctions of the conductive system. Coronary flow monitored during experiments shows the influence of drugs on the heart coronary system. Electrical activity of perfused heart has been used in studies of cardiac arrhythmias (Hwang et al. 2006, Lan et al. 2007), action potentials (APs) and T wave alternans (Qian et al. 2003), ischemic preconditioning (Bardonova et al. 2007, Fialova et al. 2009, Nygren et al. 2006), and action potential propagation (Bauer et al. 2008).

The experimental Langendorff setup used in our laboratory has been rebuilt in order to record action potentials by an optical method from local area of heart surface (Provaznik et al. 2003, Pitruzzello et al. 2007). Another option is to record optical map of electrical activity from large heart area (Laurita et al. 2000, Mills et al. 2006, Salama et al. 1987). Optical mapping is generally based on application of fluorescent voltagesensitive dye (VSD) to the examined tissue (Salama et al. 1987, Efimov et al. 1994) where it binds to a membrane of cardiac cells. VSD reacts to the local potential alterations of surrounding electric field. The dye undergoes a measurable charge shift upon excitation; the energy difference between the ground and excited states is thus sensitive to the external electric field oriented in 
direction of the shifting charge (Loew et al. 1978, Fluhler et al. 1985, Loew et al. 1985). The examined tissue is illuminated by light usually with relatively limited narrow spectra. Then, the dye emits fluorescent light of higher wavelength and amplitude proportional to the potential at the heart surface. The emitted light can be easily detected, recorded and measured.

In our experiments, the fluorescent dye di-4ANEPPS has been used. Its excitation and emission spectra contain wavelengths of visible light. The excitation spectrum corresponds to the wavelengths of blue-green light $(400-530 \mathrm{~nm})$. The emission spectrum corresponds to longer wavelengths of green-red light (550-760 nm).

There are two basic methods of emission light detection and measurement.

The first method, single-wavelength measurement of fluorescence emission, is based on detection of the emitted light for wavelengths longer than the approximate central frequency of the emission peak (610 nm for di-4-ANEPPS). However, not all information contained in the emitted light spectrum light of the dye is detected in this way. The MAP signal acquired by the single-wavelength recording setup is often deranged by motion artifact. Elimination of the motion artifact is possible by an analysis of more MAPs measured by optical mapping or by the heart movement suppression.

In case of optical mapping, the map of electrical activity of the tissue is recorded at each instant and the obtained maps are set up. The criterion of optimal function of image registration is the interrelationship between the reference and actual map (Rohde et al. 2005). The limitation of this method is that only motion artifact in a plane (2D) can be resolved. In fact, the heart moves freely in a space (3D). Thus, the heart is usually fixed to a flat glass through which the maps are recorded (Nygren et al. 2003).

The heart motion can be also reduced by calcium blocking agents. However, these blockers also affect electrophysiological properties of cardiac cells and thus cannot be generally used.

Another method of emission light detection and measurement is the dual-wavelength ratiometry which was first used in the measurements on hippocampal neurons during voltage-clamp pulses (Bullen and Saggau 1999). It is based on measurement of whole emission spectrum including its green $(510-570 \mathrm{~nm})$ and red (>590 nm) part (Himmel et al. 2006, Knisley et al. 2000, Qian et al. 2003, Tai et al. 2004). The published systems contain a dichroic mirror to split emitted light into two different light beams. Each light beam is focused onto a detector sensitive in a different range of wavelengths. Two signals corresponding to green and red light intensity are measured. Both signals contain information about the changes of membrane potential. Dualwavelength ratiometry gives the resulting action potential by the ratio calculated from green and red signals. Intensity of green light increases with the change of membrane voltage while the intensity of red light decreases. Variations in green and red fluorescence caused by photobleaching, variations in dye concentration or by motion artefacts are consistent in both detected signals. Thus, evaluating the signal ratio can be used to effectively suppress the abovementioned variations (Gross et al. 1994, Efimov et al. 1994, Knisley et al. 2000).

The present paper reviews the techniques of optical recording the monophasic action potentials (MAP) in isolated perfused rabbit heart. Two methods are reported: single-wavelength recording of fluorescence emission (Provaznik et al. 2003) and the modified dualwavelength ratiometric fluorescence emission measurement (Bardonova et al. 2008). The modification is based on improvement of the used optical path and on an advanced integrated photodetector. MAPs recorded by the above mentioned methods are described and compared. The possibility of simultaneous recording of EG and MAP and measurement of conduction velocity in the heart tissue are discussed.

\section{Methods}

\section{Animal experiments}

All animal experiments were carried out with respect to the recommendations of the European Community Guide for the Care and Use of Laboratory Animals and followed the guidelines for animal treatment approved by local authorities.

Two groups of isolated New Zealand rabbit hearts were employed in this study ( 3 animals in each). The animals were introduced into deep anesthesia by i.m. application of xylazin $(2 \mathrm{mg} / \mathrm{kg})$ and ketamin $(60 \mathrm{mg} / \mathrm{kg})$. The chest was opened, the heart with sufficiently long piece of aorta cut-off and placed in a preparation bowl with a cold $\left(5^{\circ} \mathrm{C}\right) \mathrm{Krebs}-H e n s e l e i t(\mathrm{~K}-\mathrm{H})$ solution of the following composition: $\mathrm{NaCl}: 118 \mathrm{mM}, \mathrm{NaHCO}_{3}$ : $24 \mathrm{mM}, \mathrm{KCl}: 4.2 \mathrm{mM}, \mathrm{KH}_{2} \mathrm{PO}_{4}: 1.2 \mathrm{mM}, \mathrm{MgCl}_{2}$ : $1.2 \mathrm{mM}, \mathrm{CaCl}_{2}: 1.2 \mathrm{mM}$, glucose: $5.5 \mathrm{mM}$, and Taurine: 
$10 \mathrm{mM}$. Aorta was cannulated and the heart perfused at the constant perfusion pressure $(80 \mathrm{mmHg})$ with $\mathrm{K}-\mathrm{H}$ solution. The heart was placed into the test bath, a part of the modified Langendorff setup (Novakova et al. 2000). All experiments were performed at $37^{\circ} \mathrm{C}$. The temperature was controlled in cannula and inside the bath. After isolating the heart, the experiment consisted of four consecutive phases: control perfusion, loading with the dye, dye washout and MAPs recording. Duration of control period, loading of the dye and dye washout was approximately 20 minutes. The phase of MAPs recording was usually longer depending on the respective experimental protocol (ischemia preconditioning study, pharmacological study, etc.). During the whole experiment, a function of the heart was controlled by simultaneous touch-free recordings of 3D electrograms.

\section{Voltage sensitive dye}

The fluorescent voltage-sensitive dye di-4ANEPPS (amino-naphthyl-ethenyl-pyridinium) produced by Molecular Probes, Inc., USA was used in our experiments. This dye has been proved to be the most suitable one for measurement in cardiac tissue (Fluhler et al. 1985). VSD's spectral characteristics depend on the type of tissue and the actual solvent. Absorption spectrum of excitation light ranges over spectrum of blue visible light. The excitation spectrum has almost normal distribution with maximum at $468 \mathrm{~nm}$ measured in phospholipid bilayers (Fluhler et al. 1985). The fluorescence emission spectrum ranges over the spectrum of green and red light. The emission spectrum has also almost normal distribution with maximum above $610 \mathrm{~nm}$ wavelength depending on the membrane potential. The emission spectrum shifts from left to right with increasing membrane potential. Corresponding spectral characteristics for application of di-4-ANEPPS VSD in rabbit heart tissue are shown in Figure 1.

As the VSD emission spectrum shifts with the surrounding electric field and corresponds to a local action potential, MAP can be measured by detection of light in a specific wavelength band. The easiest way of detection, which is frequently used for optical mapping, is based on sensing light with wavelengths longer than $\sim 600 \mathrm{~nm}$ (red light). This light can be filtered out by an optical filter and detected. Amplitude of the resulting signal corresponds to the area under curve of emission spectrum limited by wavelength of the optical filter from the left in spectral characteristics of the dye (Figure 1). In fact, the change of emission intensity caused by photobleaching and heart motion also affects the monitored action potential. This unwanted change can be eliminated by ratiometric fluorescence emission measurement.

\section{Recording setup}

EG signals are recorded by six silver-silver electrodes positioned orthogonally on the inner surface of the bath. EGs are amplified by bioamplifiers DAM-50 (World Precision Instruments, Inc.). MAP is recorded by a reflection optical probe which is the end of bifurcated fiber cable. The cable passes through the bath wall to the heart surface. There are two different setups for MAP measurement used in this project.

All signals are simultaneously digitized by 16 bit $\mathrm{AD}$ converters at $2 \mathrm{kHz}$ sampling rate. The signals are acquired by a LabView compatible data acquisition multifunction card PCI-6250 (National Instruments, USA).

\section{Single-wavelength measurement of fluorescence emission}

The recording setup for measurement of MAP and EG from isolated heart perfused in Langendorff setup (LPS) is shown in Figure 2 top. A $150 \mathrm{~W}$ halogen lamp with highly stable output (ripple $<0.01 \%$ ) is used as a source of excitation light. The light source contains an infrared filter to prevent the examined heart from heating and an excitation band pass filter $(506 \mathrm{~nm}+/-30 \mathrm{~nm})$. Bifurcated fiber cable is used for leading light in optical setup. All fibers are designed for wavelengths from 350 to $1100 \mathrm{~nm}$ and their diameter is $200 \mu \mathrm{m}$. The light goes through bifurcated fiber cable in both directions. Excitation light is lead from source to the heart surface through six optic fibers and emitted light is lead back from the heart to the optical sensor by a single (central) optic fiber. The emitted light goes through an emission high pass filter ( $>610 \mathrm{~nm}$ ) and then it is detected by a $\mathrm{Si}$ photodiode. The resulting electrical signal is amplified by a two-stage operational amplifier.

The usual main drawback of this method is a large motion artefact present in MAP recordings caused by relative movement of optical probe against the heart surface during the heart cycle. The heart cannot be properly mechanically fixed while the optical probe tip is only in a touch with the heart surface. One of the possible solutions avoiding chemically induced electromechanical uncoupling is the use of ratiometric recording. 


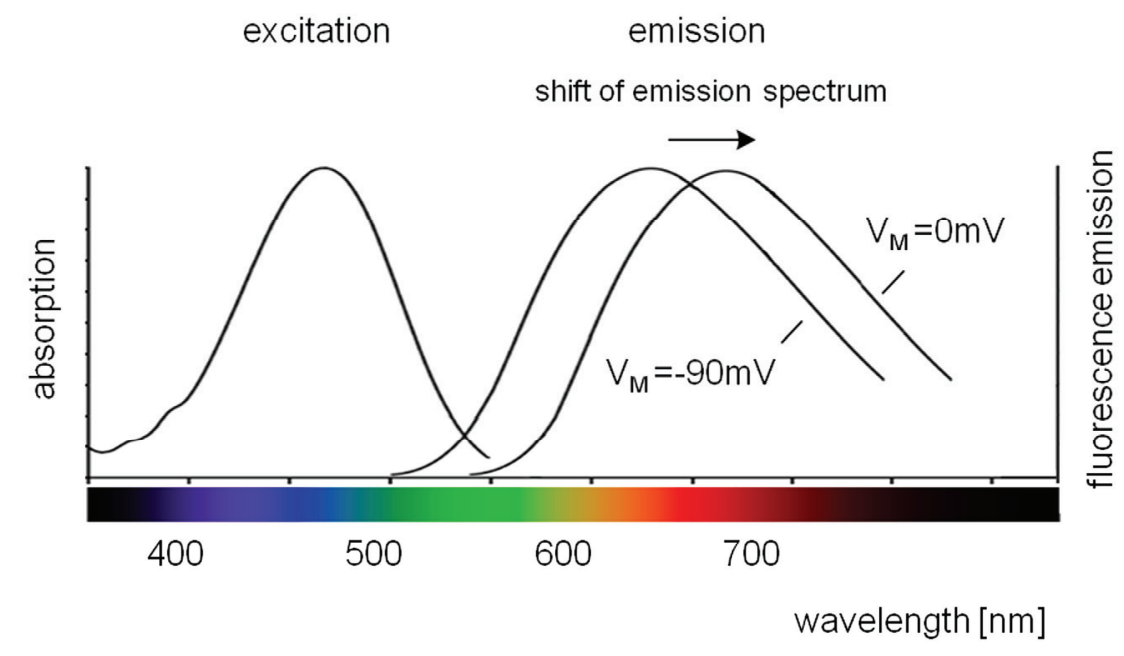

Fig. 1. Spectral characteristics of voltage-sensitive dye di-4-ANNEPS; $\mathrm{V}_{\mathrm{M}}$ - membrane voltage.
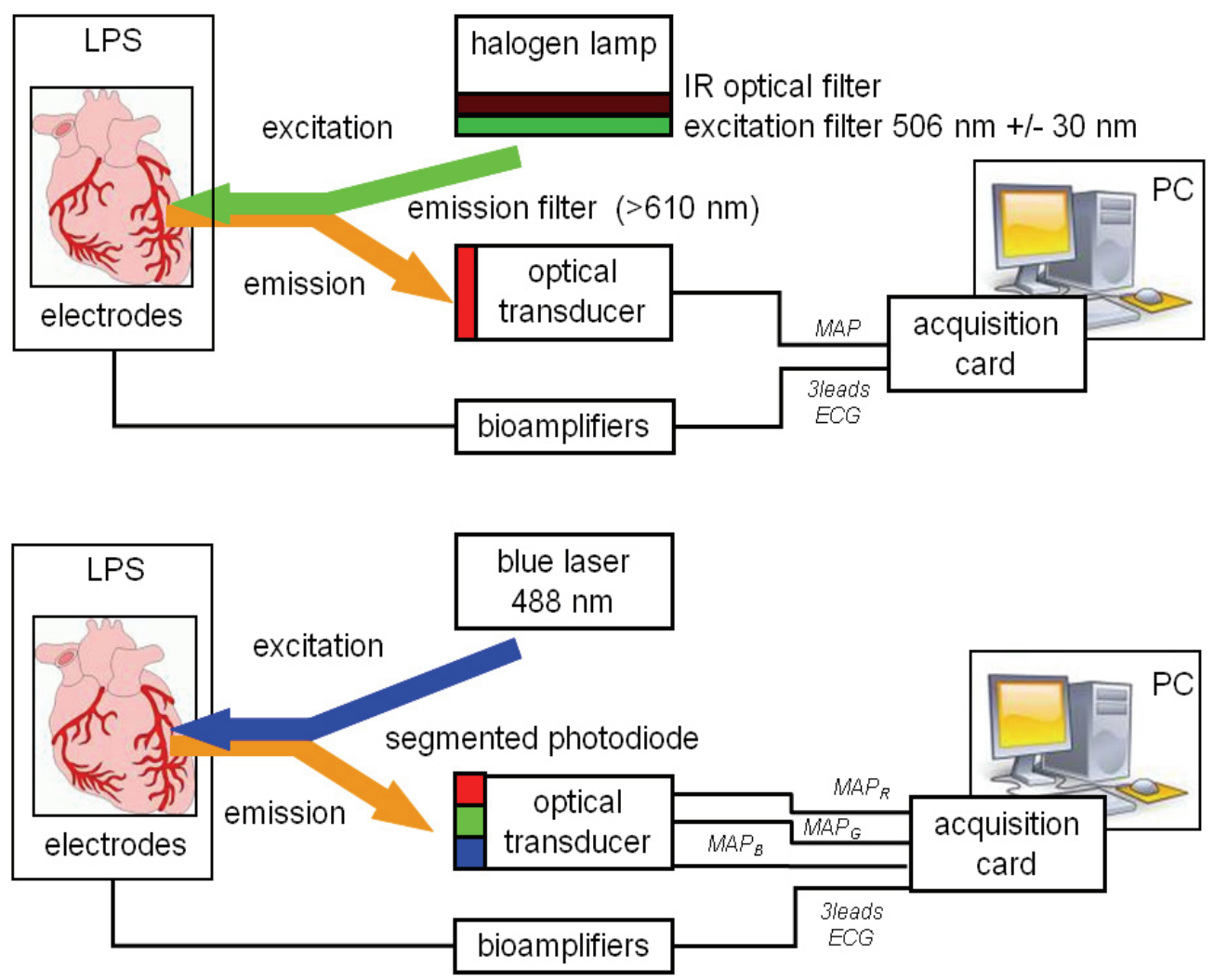

Fig. 2. Top: Block diagram of the measuring setup with three EG channels and one MAP channel recorded by single-wavelength measurement of fluorescence emission. Bottom: Block diagram of the dual-wavelength ratiometric fluorescence recording setup with three EG channels and three MAP channels. Optical transducer is composed of a segmented photodiode and three amplifiers. Output signals $M A P_{B}, M A P_{G}$ and $M A P_{R}$ correspond to blue light, green light, and red light.

\section{Ratiometric fluorescence emission measurement}

Ratiometric recording setup is based on detection of the emitted light in two narrow wavelength bands located the left and the right from the centre of emission spectrum. A segmented $\mathrm{Si}$ photodiode (Hamamatsu Photonics) was used as a detector. The photodiode has $2 \mathrm{~mm}$ diameter photosensitive area divided into three segments. Each segment represents a photodiode sensitive to visible light in a specific wavelength range. In Table 1, main spectral characteristics of the segmented photodiode are overviewed: spectral response range $(\lambda \min -\lambda \max )$ representing sensitivity area of the detector, peak sensitivity wavelength ( $\lambda$ peak) and photosensitivity. 
Table 1. Parameters of segmented photodiode (RGB sensor).

\begin{tabular}{lccc}
\hline segment & blue & green & red \\
\hline $\begin{array}{l}\lambda \min -\lambda \max [\mathrm{nm}] \\
\begin{array}{l}\lambda \text { peak [nm] } \\
\text { photosensitivity } \\
{[\mathrm{A} / \mathrm{W}]}\end{array}\end{array}$ & 0.18 & 0.23 & 0.16 \\
\hline
\end{tabular}

The recording setup is shown in Figure 2 bottom. A blue laser source with wavelength of $488 \mathrm{~nm}$ and controlled power from 0 to $20 \mathrm{~mW}$ is used as a source of excitation light. The light reflected and emitted from the heart surface passes through optic fiber to the optical electrical transducer and hits the segmented photodiode. Blue light reflected from the heart surface is converted by a photodiode to an electric signal $\mathrm{MAP}_{\mathrm{B}}$ and amplified. This signal is directly modulated by heart motion. Green and red emission light are detected by two other segments and also amplified $-\mathrm{MAP}_{\mathrm{G}}$ and $\mathrm{MAP}_{\mathrm{R}}$. $\mathrm{MAP}_{\mathrm{G}}$ and $\mathrm{MAP}_{\mathrm{R}}$, correspond to MAP affected by the motion artifact. Next, the resulting MAP signal is computed as a ratio of these two signals. Motion artifacts presented in both optical signals are divided and thus eliminated.

There are at least two main known sources of motion artefacts, which affect MAPs. First, heart motion changes the distance between the optical probe and the heart surface. Second, a number of cells under the optical fiber varies during the heart cycle. Thus, more VSD molecules are excited in contraction phase and more emission light is detected.

\section{Results}

The experiments on isolated hearts were performed in two groups. In the first group, electrical activity of the heart was followed by EG recording and MAPs were acquired by recording setup based on a single-wavelength fluorescence method. The original MAP is shown on the top trace in Figure 3. At the same time, EGs from three orthogonal leads were recorded (bottom curves).

The experiments in the second group followed the same experimental protocol with different MAP recording setup based on dual-wavelength (ratiometric) fluorescence measurement. Three optical signals $\mathrm{MAP}_{\mathrm{B}}, \mathrm{MAP}_{\mathrm{G}}$ and $\mathrm{MAP}_{\mathrm{R}}$ - are shown in Figure 4 (upper left panel) along with one of the EG channels (lower left

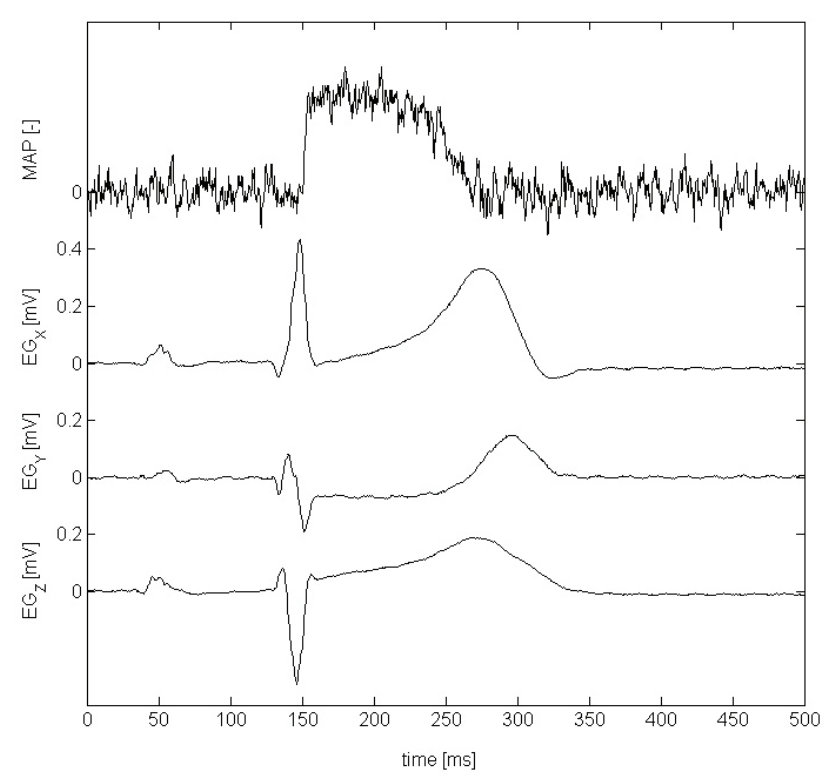

Fig. 3. Original signals from the first group of experiments. From top to bottom: MAP recorded by a single-wavelength fluorescence measurement, EG from $X, Y, Z$ axis of one heart beat.

panel). The MAP recorded at this moment is not visible in $\mathrm{MAP}_{\mathrm{G}}$ or $\mathrm{MAP}_{\mathrm{R}}$ because the practically useful component of signal is too low with respect to the noise. Three orthogonal EG signals were recorded simultaneously, one of them is shown in Figure 4. The heart was stabilized and electrogram was also stable during the analyzed time sequence. Therefore, highlighting of a useful component part of signal is possible by averaging the optical signals.

EGs were processed by R-peak detector to determine R-R intervals. The onset of cardiac cycle was established as $30 \%$ of $\mathrm{R}-\mathrm{R}$ interval before $\mathrm{R}$ peak. Seventy consecutive cardiac cycles were detected. Then, EG and MAP during cardiac cycle were obtained by sample-by-sample averaging.

In order to minimize the putative effect of timerelated changes of excitation, the averaged $\mathrm{MAP}_{\mathrm{G}}$ and averaged $\mathrm{MAP}_{R}$ were normalized by averaged $\mathrm{MAP}_{B}$ (Figure 4, top right panel). The upstroke of MAP obtained in $\mathrm{MAP}_{\mathrm{R}}$ is positive and upstroke of MAP obtained in $\mathrm{MAP}_{\mathrm{G}}$ is negative (indicated by arrows in Fig. 4). On the other hand, motion artifact has the same trend in both signals (dotted arrows in Fig. 4). The resulting MAP is emphasized and the motion artifact is reduced by the ratio of $\mathrm{MAP}_{\mathrm{R}}$ and $\mathrm{MAP}_{\mathrm{G}}$. Maximum amplitude of MAP is normalized, for $100 \mathrm{mV}\left(\mathrm{MAP}_{\mathrm{n}}\right)$. (Fig. 4, upper trace of the right bottom panel; the lower trace is the averaged electrogram). In $\mathrm{MAP}_{n}$, there is an evident residue of the motion artifact at time $130-200 \mathrm{~ms}$. This 

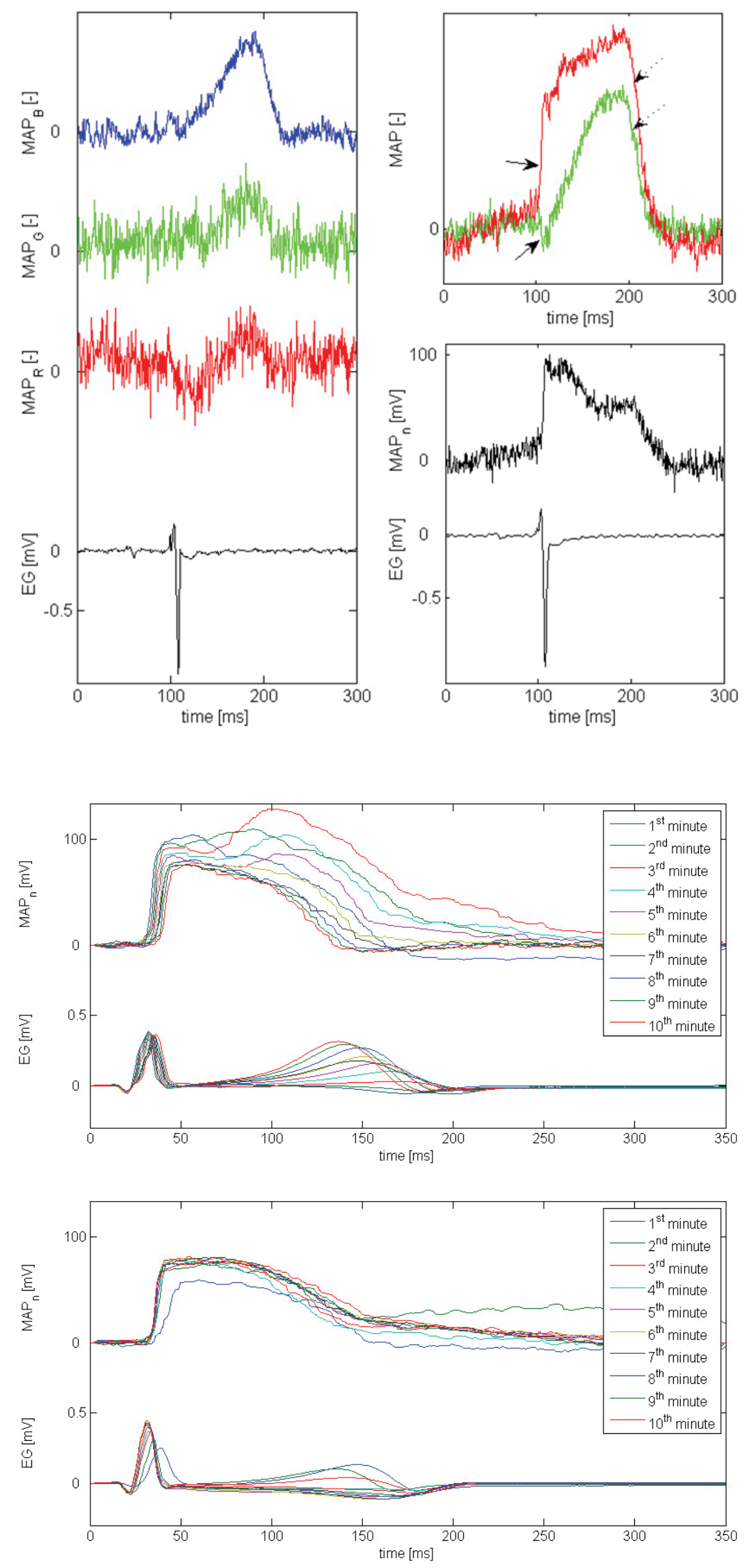

Fig. 4. Left panel: Simultaneous original recording of $M A P_{B}, M_{A} P_{G}$ and $\mathrm{MAP}_{R}$ (raw data) corresponding to detected blue, green and red light and one lead of EG signal during one heart cycle. Right panel (above): averaged $\mathrm{MAP}_{G}$ and averaged $M A P_{R}$ (normalized by $M A P_{B}$ ). Right panel (bottom): Resulting normalized $M{ } P_{n}$ obtained by ratiometry and averaged electrogram ( $T$ wave not visible).

Fig. 5. Top: The simultaneous recording of EGs and $\mathrm{MAP}_{n} s$ during ten minutes of ischemia period. Bottom: The simultaneous recording of EGs and MAPs during ten minutes of reperfusion period. 
distortion of MAPn is probably evoked by overlap of spectral response ranges of green and red segment of segmented photodiode. Other possible reason of this distortion is the fact that the wavelength range of green part of the detected emission light has to be shorter than the wavelength of maximum of emission spectrum. In analogy, the wavelength range of red portion of detected emission has to be longer than the wavelength of maximum of emission spectrum. The wavelength of the spectrum maximum is different for various values of MAP $_{n}$. The MAP ${ }_{n}$ recorded in this way is apparent with clear upstroke and measurable duration.

Recording setup for fluorescent measurement in our experiments was not calibrated due to lack of a proper calibration method. MAP is usually presented as a relative signal. Another approach is to normalize MAP to voltage range of $100 \mathrm{mV}$ (Salama et al. 2005).

In both types of experiments, EG signals were recorded from three leads during the whole experiment. MAPs were measured from a chosen point on the left ventricle surface. Both EG and MAP signals were recorded simultaneously during up to six consecutive phases of the experiment.

\section{Effects of ischemia and reperfusion}

In the first group of experiments, MAP was recorded in good quality for at least 60 minutes. The quality of MAP recordings obtained by singlewavelength fluorescent measurement was discussed earlier (Novakova et al. 2008). MAP is distorted by motion artifacts, which are represented by many different shapes and locations on the recorded signal. Usually, the beginnings of MAP are artifacts-free and could be used for analysis together with EG signal. The heart depolarization process during experiment can be followed. The study of repolarization from MAP is possible only partly.

The uninterrupted long-lasting MAP and EG recordings may be used for the measurement of velocity of impulse conduction through isolated rabbit hearts perfused according to Langendorff. The velocity of impulse conduction was studied during preconditioning experiments (Kolarova et al. 2009). A global ischemia was introduced by stopping the perfusion for 10 minutes and MAPs and EG signals were continuously and simultaneously recorded. Then, 10 minutes of reperfusion followed. These two ischemia-reperfusion periods were repeated three times.

The beginning of QRS complex and MAP upstroke was detected. The distance from the earliest QRS deflection to the MAP upstroke was defined as the activation time (AT) (Franz et al. 1987). AT corresponds to the velocity of impulse conduction through ventricular tissue during depolarization from bundle branches to the position of optical probe on the heart surface.

All three periods of ischemia and reperfusion were analyzed. In each minute of the experimental periods, the average MAP and EG of cardiac cycles were calculated to suppress noise. Then, MAPs and EG cycles were positioned to the onset of QRS complex, which represents the beginning of AT. All averaged cycles were normalized so that the first MAP at the beginning of ischemia period was set to $100 \mathrm{mV}\left(\mathrm{MAP}_{\mathrm{n}}\right)$.

An example of simultaneously recorded MAP and EG signals from a single experiment is shown in Figure 5. The progress of ischemia is shown on the top panel and progress of reperfusion is shown at the bottom of the figure.

$\mathrm{MAP}_{\mathrm{n}}$ decayed during ischemia period. As discussed above, the recorded MAP signal contained noise and motion artifacts. However their characteristics varied from experiment to experiment. In experiment documented in Figure 5, the large motion artefact was noticed from the first to the sixth minute with maximum around the third minute of ischemia. MAP did not contain the motion artefact from the seventh minute. The magnitude of depolarization upstroke decreased in time most probably due to influence of ischemia and photobleaching. MAP duration shortened and AT prolonged progressively and almost linearly. Prolongation of AT corresponds to deceleration of spread of depolarization, which can be clearly recognized from EG. During the second and the third period of repeated ischemia, AT prolonged and the value of depolarization further decreased.

During reperfusion period, magnitude of depolarization upstroke of MAP recorded in the first minute is obviously lower and AT is slightly shorter than AT in the last minute of ischemia. Then, AT quickly shortened. It is caused by fast recovery of perfusion that restores electrical parameters of the tissue, particularly velocity of propagation of depolarization.

The change of conduction velocity results in a change of shape of EG records as discussed earlier (Bardonova et al. 2008). The changes of MAP configuration brought about by preconditioning were published (Kolarova et al. 2009). 


\section{Discussion}

Monophasic action potentials may be efficiently recorded by optical methods using fluorescence of voltage-sensitive dyes. Main drawbacks of these methods are motion artefacts present in the recorded signals due to unpredictable relative movement of the optical probe with the heart surface.

Two optical recording setups were designed and tested. First one - a single wavelength recording setup consists of a wide spectrum halogen lamp light source, a set of optical filters to excite voltage-sensitive dye in a narrow spectral band, an optical filter to suppress the excitation light reflected from the heart tissue, a lownoise photodiode detector and a low-noise biological amplifier. The system is completed by a multi-purpose acquisition card and a personal computer for digitization of the recorded signals. Recording setup is technologically uncomplicated and provides high-quality signals. Decent suppression of signals must be expected due to the use of optical filters. The system does not offer any possibility to suppress motion artefacts present in the recorded MAPs. This disadvantage may be limited by mechanical constraints or electro-mechanical decouplers.

The second optical setup designed in our laboratory uses a segmented photodiode sensor for dualwavelength ratiometric measurement. In general, ratiometric methods are effective in suppressing the motion artefacts. However, they usually require two individual light sensors for emission detection in different spectral bands. The reported system does not require any beam splitter and exploits a package of three photodiode sensors operating in three different spectral bands. Such system requires the use of a blue laser to avoid optical filters. Two of the photodiodes server to acquire emission for ratiometry, the third photodiode sensitive in blue light band corresponds to range of excitation light. The signal recorded from the third sensor may be used for detection of reflected excitation light, which is modulated by the heart motion.

The motion artifact is caused by movement of the heart against the probe and by contraction of the heart under the probe. While the motion of the whole heart may be eliminated by mechanical constraints, the contraction of the cells and movement of the tissue is difficult to control. The epicardial cells contract bellow the optic fiber and the number of excited molecules of dye is changing during the cycle. In the course of contraction, the number of excited dye molecules increases as does the intensity of the emission light. The maximum contraction coincides approximately with the centre of MAP and is the source of maximum motion artifact on MAP signal.

The proposed ratiometric acquisition system includes the segmented photodiode, which replaces dichroic mirror and individual detectors. The system is extremely flexible and allows taking optical recording from a number of sites on the heart surface during the experiment. Furthermore, it allows using of low intensity source for excitation of VSD. The system may be used for other applications employing other fluorescent dyes. For example, measurements of action potentials and of calcium transients using two different dyes are possible. The system enables simultaneous measurement of emission light in three different spectral bands. Thus, up to three different kinds of responses may be recorded.

There are several issues in design of our dualwavelength ratiometric optical recording system which should be addressed. In this study, we used relatively high number of averaged heart cycles to reach sufficiently high signal-to-noise ratio. However, such high number of averaged repetitions restrains more detailed studies of MAP waveform alterations. A newly proposed setup solves this problem by increasing intensity of excitation light. Thus, it provides sufficient signal-to-noise ratio with averaging of less than 15 heart cycles.

\section{Conflict of Interest}

There is no conflict of interest.

\section{Acknowledgements}

This work was supported by the grant projects of the Grant Agency GACR 102/07/1473, GACR 102/07/P521, GACR 102/09/H083, MSM 0021622402, and MSM0021630513.

\section{References}

BAUER S, FRUHNER S, ROMERO I, ENGEL H, BAR M: Propagation of electrical excitation in isolated rabbit hearts: Influence of stimulation protocol and spatial coupling. Comput Cardiol 35: 299-302, 2008. 
BARDONOVA J, PROVAZNIK I, NOVAKOVA M, SEKORA J, SVRCEK M: Statistical analysis in complex-valued wavelet analysis of voltage-sensitive dye mapping. Comput Cardiol 34: 101-104, 2007.

BARDONOVA J, PROVAZNIK I, NOVAKOVA M, NOGOVA K, SEKORA J: New recording setup for ratiometric recording of action potentials by optical means. Comput Cardiol 35: 1085-1088, 2008.

BULLEN A, SAGGAU P: High-speed, random-access fluorescence microscopy: II. Fast quantitative measurements with voltage-sensitive dyes. Biophys J 76: 2272-2287, 1999.

EFIMOV I, HUANG D, RENDT J, SALAMA G: Optical mapping of repolarization and refractoriness from intact hearts. Circulation 90: 1469-1480, 1994.

FIALOVA K, KOLAROVA J, PROVAZNIK I, NOVAKOVA M: Model of preconditioning in guinea pig and rabbit isolated hearts loaded with voltage-sensitive dye di-4-ANEPPS. Comput Cardiol 36: 529-532, 2009.

FLUHLER E, BURNHAM V, LOEW L: Spectra, membrane binding, and potentiometric responses of new charge shift probes. Biochemistry 24: 5749-5755, 1985.

FRANZ MR, BARGHEER K, RAFFLENBEUL W, HAVERICH A, LICHTLEN PR: Monophasic action potential mapping in human subjects with normal electrocardiograms: direct evidence for the genesis of the $\mathrm{T}$ wave. Circulation 75: 379-386, 1987.

GROSS E, BEDLACK RS, LOEW LM: Dual-wavelength ratiometric fluorescence measurement of the membrane dipole potential. Biophys J 67: 208-216, 1994.

HIMEL H, KNISLEY S: Imaging of cardiac movement using ratiometric and nonratiometric optical mapping: effects of ischemia and 2, 3-butaneodione monoxime. IEEE Trans Med Imaging 25: 122-127, 2006.

HWANG GS, HAYASHI H, TANG L, OGAWA M, HERNANDEZ H, TAN A, LI H, KARAGUEUZIAN H, WEISS J, LIN SF, CHEN PS: Intracellular calcium and vulnerability to fibrillation and defibrillation in Langendorff-perfused rabbit ventricles. Circulation 114: 2595-2603, 2006.

KNISLEY SB, JUSTICE RK, KONG W, JOHNSON PL: Ratiometry of transmembrane voltage-sensitive fluorescent dye emission in hearts. Am J Physiol 279: H1421-H1433, 2000.

KOLAROVA J, JANOUSEK O, NOVAKOVA M, FIALOVA K, PROVAZNIK I: Influence of ischemia and reperfusion duration on left ventricular depolarization in isolated rabbit hearts registered by optical method. Comput Cardiol 36: 525-528, 2009.

LAN DZ, POLLARD AE, KNISLEY SB, FAST VG: Optical mapping of $\mathrm{V}_{\mathrm{m}}$ and $\mathrm{Ca}_{\mathrm{i}}{ }^{2+}$ in a model of arrhythmias induced by local catecholamine application in patterned cell cultures. Pflügers Arch 453: 871-877, 2007.

LAURITA K, SINGAL A: Mapping action potentials and calcium transients simultaneously from the intact heart. $A m J$ Physiol 280: H2053-H2060, 2001.

LOEW L, COHEN L, SALZBERG B, OBAID A, BEZANILLA F: Charge-shift probes of membrane potential. Characterization of aminostyrylpyridinium dyes on the squid giant axon. Biophys J 47: 71-77, 1985.

LOEW L, BONNEVILLE GW, SUROW J: Charge shift probes of membrane potential. Theory. Biochemistry 17: 4065 4071, 1978.

MILLS W, MAL N, FORUDI F, POPOVIC Z, PENN M, LAURITA K: Optical mapping of late myocardial infarction in rats. Am J Physiol 290: H1298-H1306, 2006.

NOVAKOVA M: Effects of sigma receptor ligand BD737 in rat isolated hearts. Scripta Med 80: 255-262, 2007.

NOVAKOVA M, BARDONOVA J, PROVAZNIK I, TABORSKA E, BOCHORAKOVA H, PAULOVA H, HORKY D: Effects of voltage sensitive dye di-4-ANEPPS on guinea pig and rabbit myocardium. Gen Physiol Biophys 27: 45-54, 2008.

NOVAKOVA, M. MOUDR J, BRAVENY P: A modified perfusion system for pharmacological studies in isolated hearts. Analysis of Biomedical Signals and Images 15: 162-164, 2000.

NYGREN A, KONDO C, CLARK R, GILES W: Voltage-sensitive dye mapping in Langendorff perfused rat hearts. Am J Physiol 284: H892-H902, 2003.

NYGREN A, BACZKO I, GILES W: Measurements of electrophysiological effects of components of acute ischemia in Langendorff-perfused rat hearts using voltagesensitive dye mapping. J Cardiovasc Electrophysiol 17: S113S123, 2006.

PITRUZZELLO A, KRASSOWSKA W, IDRISS S: Spatial heterogeneity of the restitution portrait in rabbit epicardium. Am J Physiol 292: H1568-H1578, 2007. 
PROVAZNIK I. NOVAKOVA M, VESELÝ Z, BLAHA M, CHMELAR M: Electro-optical recording system for myocardial ischemia studies in animal experiments. Comput Cardiol 30: 573-576, 2003.

ROHDE G, DAWANT B, LIN S: Correction of motion artifact in cardiac optical mapping using image registration. IEEE Trans Biomed Engn 52: 338-341, 2005.

TAI D, CALDWELL B, LEGRICE I, HOOKS D, PULLAN A, SMAILL B: Correction of motion artifact in transmembrane voltage-sensitive fluorescent dye emission in hearts. Am J Physiol 287: H985-H993, 2004.

SALAMA G, CHOI B, AZOUR G, LAVASANI M, TUMBEV V, SALZBERG B, PATRICK M, ERNST L, WAGGONER A: Properties of new, long-wavelength, voltage-sensitive dyes in the heart. J Membr Biol 208: 125-140, 2005.

SALAMA G, LOMBARDI R, ELSON J: Maps of optical action potential and NADH fluorescence in intact working hearts. Am J Physiol 252: H384-H394, 1987.

QIAN YW, SUNG R, LIN SF, PROVINCE R, CLUSIN W: Spatial heterogeneity of action potential alternans during global ischemia in the rabbit heart. Am J Physiol 285: H2722-2733, 2003.

VALENTIN J, HOFFMANN P, CLERCK FD, HAMMOND T, HONDEGHEM L: Review of the predictive value of the Langendorff heart model (screenit system) in assessing the proarrhythmic potential of drugs. $J$ Pharmacol Toxicol Methods 49: 171-181, 2004.

WALL T, DALTONA J, WOLFEA M, HIGDONA N, CLARKA M, GAUVINA D, BAIRDA T: Effects of cisapride, sotalol and haloperidol in a Langendorff assay and comparison to herg, purkinje fiber and in vivo telemetry assays. J Pharmacol Toxicol Methods 56: 1741-1754, 2007. 